\title{
Two-Dimensionally Spread Multicarrier CDMA Ranging Signals With PAPR Lower Than 3dB
}

\author{
Mariano Vergara $^{(1)}$, Felix Antreich ${ }^{(1)}$, Gonzalo Seco-Granados ${ }^{(2)}$ \\ (1) German Aerospace Center (DLR), Germany \\ (2) Universitat Autónoma de Barcelona (UAB), Spain*
}

\begin{abstract}
In this work we propose a methodology to design two-dimensional spreading codes for a multicarrier CDMA (code division multiple access) system with minimal multiple access interference (MAI) and minimal peak-to-average-power-ratio (PAPR). In particular, we minimise the cross-correlation peak between two two-dimensionally spread CDMA signals, with the side-constraints that both multicarrier signals must have a PAPR lower than $3 \mathrm{~dB}$, and autocorrelation side-peaks lower than a certain value. The underlying principle of this work is that the users of a CDMA system need not be allocated exactly the same pulse shape, and that by assigning different pulse shapes, it is possible to increase the decorrelation among interfering signals. The resulting multi-dimensional non-linear optimization problem is solved by inspection of all possible solutions, as we show that the feasible region is a discrete countable set, whose size is reasonably small. The mathematical tool used in the solution of this paper consists of some radar pulse compression codes called Huffman codes.
\end{abstract}

\section{INTRODUCTION}

In order to inherit the advantages of both code division multiple access (CDMA) and multicarrier (MC) systems, several combinations of these two techniques have been developed over the course of the last two decades [1], [2]. The first proposed scheme was the so-called MC-CDMA (multi-carrierCDMA) [3], which can be considered the dual technique of the DS-CDMA (direct-sequence-CDMA) technique, as the spreading codes are sequences in the frequency-, rather timedomain. Another variant is the MC-DS-CDMA technique, where one or more low-rate DS-CDMA signals modulate multiple subcarriers [1]. A hybrid technique between DSCDMA and MC-CDMA that performs spreading in both domains, frequency and time, has also been proposed [4]. Such a multicarrier CDMA signal has been denoted in several way: doubly spread or two-dimensionally spread CDMA, or also CDMA with time-frequency spreading. This technique can also be seen as MC-CDMA system whose spreading codes vary cyclically over time, or equivalently, as MC-DS-CDMA system with code-defined complex gains on each subchannel. According to the latter interpretation, some authors have considered this CDMA technique with two-dimensional spreading a variant or a generalization of the MC-DS-CDMA scheme [5], [6]. The OFCDM modulation [7] proposed for the downlink transmission in $4 \mathrm{G}$ system is another label for the same

* The work of Gonzalo Seco is supported by the Spanish Government under project TEC2011-28219 transmission technique. CDMA signals with time-frequency spreading are very promising due to their flexibility and these signals are particularly suitable for dynamical resources allocation schemes [6]. Nevertheless, as other multicarrier signals, CDMA signals with two-dimensional spreading suffer from high peak-to-average-power-ratio (PAPR) values. High PAPR values jeopardise the power efficiency of the system, as a certain power input back-off is needed to avoid non-linear distortions caused by the transmitter's high power amplifier (HPA).

Moreover, user separation in doubly-spread CDMA systems is more problematic than in a CDMA system. Indeed, the division of the available bandwidth in $\mathrm{M}$ sub-bands make the chip duration $M$ times longer, and thus the time-domain spreading codes last $\mathrm{M}$ time longer. As a consequence of that, the time to perform a correlation over a data-symbol, and hence the time to separate users, is $M$ times longer. In order to separate the users of a doubly-spread CDMA system in time intervals comparable to the time intervals required by a DS-CDMA system with the same resources, a doublyspread signal must rely on frequency- as well time-domain for user separation. Although the design of time-domain spreading codes has been widely investigated, the design of frequencydomain spreading codes minimising the multiple access interference (MAI) of an asynchronous multicarrier CDMA system is an open field of research, e.g. [8], and it is a very challenging problem. This problem becomes even more difficult if timeand frequency-domain spreading codes. To our knowledge, no theoretical bound on the auto- and cross-correlations properties of asynchronous two-dimensional spread CDMA signals has been derived yet, in the same fashion as it was made for DSCDMA signals [9], [10]. Literature on two-dimensional spread CDMA signals [6] [5] has so far been mostly focused on the design of tree-structured orthogonal variable spreading factor (OVSF) codes and on the several code allocation strategies for the downlink channel (synchronous transmission). The objective of these authors was the maximization of the system capacity through the minimisation of the MAI. The minimization of the MAI is important for any spread spectrum systems, yet some wireless systems may have different priorities as mobile communication systems. For example, in case of global navigation satellite systems (GNSS) a totally different scenario from the ones targeted by the above mentioned literature is contemplated. First of all, inter-system MAI is not the main factor limiting the system performance of a GNSS, due to a 
significantly smaller number of users (satellites) sharing the same radio resource. Beside being small, the number of users (satellites) sharing the radio resource is also constant, and dynamical code allocations are neither practical nor particularly helpful. Furthermore, due to the limited power available on satellite payloads and to the small antenna gains of GNSS receivers, power efficiency plays a much more dominant role than in terrestrial wireless downlink transmission. On these grounds, in wireless systems as GNSS the minimization of the power losses arising from the distortion caused by the transmitter's high-power-amplifier (HPA) has higher priority than in other communication systems. To this aim, the PAPR of the transmitted signal must be kept very low, i.e. comparable to the PAPR of a single carrier signal. The data rate to be supported by a GNSS is significantly lower (of the order of Kbits/sec) than the data rates supported by other wireless communication systems using the same bandwidth. This makes the problem of reducing the PAPR of multicarrier signals used for GNSS different and for certain aspects even easier than than the equivalent problem arising in multicarrier communication systems. Indeed, in multicarrier communication systems the aim is to minimise the PAPR keeping at the same time a relatively high data rate; in a wireless systems that is primarily meant for ranging, this constraint is not so strict. These considerations, as well as this study, concern any localization application based on multicarrier transmission working with a reduced power supply and a limited constant number of users sharing the same radio-frequency resource.

In this paper we tackle the problem of two-dimensional spreading codes design for an asynchronous The contribution of this work consists in providing a methodology for the design of frequency spreading codes which minimise the MAI and which at the same time contain the PAPR of the signal to values smaller than $3 \mathrm{~dB}$. The upper bound of the PAPR is $3 \mathrm{~dB}$ because this is an indirect result of the application to this problem of some interesting results developed for radar pulse compression [11]. The core idea of this paper stems from the consideration that there is no sound technical reason for which all users should be assigned exactly the same pulse shape, as long as several system performance requirements are met (i.e. ranging accuracy, tracking robustness, max PAPR). The method we propose is based on a new application of interesting theoretical results of radar pulse-compression theory. The presented approach is valid for an arbitrary finite number of subcarriers and it has the advantage of being scalable. In the considered scenario only two interfering users have been considered. We show that the resulting multi-dimensional nonlinear optimization problem can be solved by inspection of all possible solutions, as the feasible region is a discrete countable set with a reasonably small cardinality.

\section{SySTEM MODEL}

\section{A. Signal model}

The ranging signal assigned to a generic user $u$ in a CDMA system with two-dimensional spreading can be written as

$$
s_{u}(t)=\sqrt{P} \sum_{n=0}^{N-1} \sum_{m=0}^{M-1} c_{n, m}^{u} g\left(t-n T_{c}\right) e^{j 2 \pi m \Delta_{f} t}
$$

where $P$ is the signal power, $g(t)$ is the finite energy prototype filter $\left(\int_{-\infty}^{\infty}|g(t)|^{2} \mathrm{~d} t=T_{c}\right), T_{c}$ is the chip duration, $T=N T_{c}$ is the information symbol time, and the term $\Delta_{f}$ is the frequency separation between the subcarriers. The product $\Delta_{f} T_{c}$ indicates how dense the frequency-time grid is. Weyl-Heisenberg theory [12] determines how to choose this product with respect to the prototype filter. In this study, we assume an ideally rectangular prototype filter and an integer product $\Delta_{f} T_{c}$, as in OFDM (orthogonal frequency division multiplex) signals. This choice enables the to formulate the PAPR reduction problem in a easier mathematical form. The matrix

$$
\mathbf{C}^{u}=\left(\begin{array}{ccccc}
c_{0,0}^{u} & c_{0,1}^{u} & \ldots & \ldots & c_{0, M-1}^{u} \\
\vdots & \ddots & \vdots & \ddots & \vdots \\
c_{n, 0}^{u} & \ldots & c_{n, m}^{u} & \ldots & c_{n, M-1}^{u} \\
\vdots & \ddots & \vdots & \ddots & \vdots \\
c_{N-1,0}^{u} & c_{N-1,1}^{u} & \ldots & \ldots & c_{N-1, M-1}^{u}
\end{array}\right) \in \mathbb{C}^{N \times M}
$$

is called spreading matrix and the superscript $u$ indicates that this matrix identifies the $u$-th user. We assume that the spreading matrix can be partitioned in such a way that each element can be written as

$$
c_{n, m}^{u}=c_{T}^{u}[n] \cdot c_{F}^{u}[m]
$$

with $\left\{c_{T}^{u}[n]\right\}, n=0, \ldots, N-1$ the time-domain spreading code and $\left\{c_{F}^{u}[m]\right\}, m=0, \ldots, M-1$ the frequency-domain spreading code. The codes are normed in this way:

$$
\begin{aligned}
\left\|\mathbf{c}_{T}^{u}\right\|_{2}^{2} & =N \\
\left\|\mathbf{c}_{F}^{u}\right\|_{2}^{2} & =1
\end{aligned}
$$

such that the signal power is $P$. We consider constant envelope time-domain spreading codes, i.e.

$$
\left|c_{T}^{u}[n]\right|=1 \quad \forall n .
$$

which amounts to state that the time-domain spreading codes are polyphase codes.

Condition (3) decouples the design of frequency- and timespreading codes. Since time-domain spreading codes have no effect on the PAPR of the signal (1), they can be used exclusively to minimise MAI. On the other hand, frequencydomain spreading codes are can be used to jointly minimise MAI and PAPR. In this paper, we take care only of the design of the frequency-domain spreading codes, as the design of time-domain spreading codes has already been extensively analysed (e.g. [10]). In the problem at hands, time-domain spreading codes can be chosen from any set of codes with excellent correlation properties, as for example Zadoff-Chu codes [13].

For the sake of clarity, we remind the reader that dispersion takes place exclusively in the frequency domain. The spreading operation is carried out in two stages: one is performed in the time-domain and the other in frequency-domain. The relative 
order of the two stages is irrelevant from a modelling point of view. The spreading in time is performed by a multiplication of the data modulated stream (in this paper there is no data) signal by a train of symbols (chips) transmitted at a much higher rate than the data stream, and whose phases are dictated by the time-domain spreading code. The spreading in frequency is performed by replicating the data modulated stream on different sub-carriers, with complex gains (amplitudes and phases), dependent on the frequency-domain spreading code. In the following the time- and frequency-domain spreading codes will be shortly indicated with time codes and frequency codes.

When a multicarrier signal is used to data transmission, the property that it is required from its autocorrelation function concerns only time instants that are integer multiples of the symbol time, in our model equal to a integer multiple of the inverse of the frequency separation between the subcarriers. In particular, the autocorrelation function must be zero at these time instants, so that inter-symbol interference (ISI) does not occur. For all other time instants, the autocorrelation function of a multicarrier signal as (1) is not relevant from a strictly data-transmission point of view, provided that the orthogonality of the subcarriers is maintained. On the contrary, when a multicarrier signal is used for ranging all values of its autocorrelation are of interest. Particularly important are the peaks of the autocorrelation function and the values around the main peak of the autocorrelation function. The formers determine the robustness of the tracking (and coarse acquisition) process, and the latter the accuracy of the ranging. At these values of the autocorrelation function it is not possible to assume orthogonality among the subcarriers, if the prototype filters are not strictly band-limited, as it is the case of this study. Same holds for the cross-correlation function. As a consequence of that, the MAI between two or users consists of two terms:

1) the interference created by other users transmitting at the same subcarrier frequency,

2) the interference created by non-reference users transmitting at different subcarrier frequencies.

The first term is common to single- as well multi-carrier systems. The second term is caused by ICI and is due to the nature of multicarrier transmission. Since all values of the auto- and cross-correlation function are needed, and not only the one located at multiple integers of the symbol time, the second term appears even though the users are synchronised. Thus, there is not a big difference between synchronous and asynchronous transmission for the MAI of a ranging MCCDMA, as instead it happens for data communication systems. The study of the MAI in a MC-CDMA (and two-dimensionally spread) ranging system is similar to the MAI found in an asynchronous MC-CDMA communication system [14]. If the channel is assumed ideal, the cross-correlation function at the receiver side between the signal of user $u$ and the signal of user $v$ is

$$
\begin{aligned}
& R_{s_{u}, s_{v}}(\tau)=\frac{1}{T} \int_{T} s_{u}(t) s_{v}^{*}(t+\tau) \mathrm{d} t= \\
& \sum_{m_{1}=0}^{M-1} \sum_{m_{2}=0}^{M-1} c_{F}^{u}\left[m_{1}\right]\left(c_{F}^{v}\left[m_{2}\right]\right)^{*} e^{-j 2 \pi m_{2} \Delta_{f} \tau} \\
& \quad \times \sum_{n=0}^{N-1} \sum_{d=-\infty}^{\infty} c_{T}^{u}[n]\left(c_{T}^{v}\right)^{*}[n+d] \\
& \quad \times \frac{1}{T} \int_{T} g(t) g\left(t-d T_{c}+\tau\right) e^{j 2 \pi\left(m_{1}-m_{2}\right) \Delta_{f} t} \mathrm{~d} t .
\end{aligned}
$$

This expression contains terms without inter-channel interference $\left(m_{1}=m_{2}\right)$ and terms representing inter-channel interference $\left(m_{1} \neq m_{2}\right)$. Inter-channel interference terms arise because the subcarriers are no more orthogonal for time-delays different from the chip duration $\left(\tau \neq T_{c}\right)$. If the delay $\tau$ differs by a small amount from an integer multiple of $T_{c}$, i.e. if:

$$
\min _{d \in \mathbb{Z}}\left\{\left|\tau-d T_{c}\right|\right\} \ll 1
$$

then the ICI terms are small and we can approximate (7) with the only inter-channel-interference-free terms:

$$
\begin{aligned}
& R_{s_{u}, s_{v}}(\tau) \approx \sum_{m=0}^{M-1} c_{F}^{u}[m]\left(c_{F}^{v}\right)^{*}[m] e^{-j 2 \pi m \Delta_{f} \tau} \\
& \sum_{d=-\infty}^{\infty} \frac{\sum_{n=0}^{N-1} c_{T}^{u}[n]\left(c_{T}^{v}\right)^{*}[n+d]}{N} \frac{1}{T_{c}} \int_{-\frac{T}{2}}^{\frac{T}{2}} g(t) g\left(t-d T_{c}+\tau\right) \mathrm{d} t \\
& =\sum_{d=-\infty}^{\infty} R_{\mathbf{c}_{T}^{u}, \mathbf{c}_{T}^{v}}[d] R_{g}\left(\tau-d T_{c}\right) \tilde{R}_{\mathbf{c}_{F}^{u}, \mathbf{c}_{F}^{v}}(\tau)
\end{aligned}
$$

where

$$
\begin{gathered}
R_{\mathbf{c}_{T}^{u}, \mathbf{c}_{T}^{v}}[d]=\frac{1}{N} \sum_{m=0}^{N-1} c_{T}^{u}[m]\left(c_{T}^{v}\left[(m+d)_{\bmod N}\right]\right)^{*}, \\
\tilde{R}_{\mathbf{c}_{F}^{u}, \mathbf{c}_{F}^{v}}(\tau)=\sum_{m=0}^{M-1} c_{F}^{u}[m]\left(c_{F}^{v}[m]\right)^{*} e^{j 2 \pi m \Delta_{f} \tau}, \\
R_{g}(\tau)=\int_{-\infty}^{\infty} g(t) g(t+\tau) \mathrm{d} t
\end{gathered}
$$

are respectively the periodic cross-correlation between the time codes, a quantity that we call spectral correlation between the frequency codes, and lastly the autocorrelation of the rectangular window function. The integration interval $\left[-\frac{T}{2} ; \frac{T}{2}\right]$ can be approximately considered infinite when $T>>T_{c}$, i.e. when the time codes are very long. Note that the approximation (8) becomes more accurate as the frequency separation is increased, as the signal (16) becomes closed to a FDMA (frequency division multiplex access) signal.

We define the pulse autocorrelation of the user $u$ as:

$$
R_{u, u}^{p u l s e}(\tau)=R_{g}(\tau) \tilde{R}_{\mathbf{c}_{F}^{u}, \mathbf{c}_{F}^{u}}(\tau)
$$

and analogously, the cross-correlation between the pulses of user $u$ and user $v$ :

$$
R_{u, v}^{p u l s e}(\tau)=R_{g}(\tau) \tilde{R}_{\mathbf{c}_{F}^{u}, \mathbf{c}_{F}^{u}}(\tau)
$$


Since $\tilde{R}_{\mathbf{c}_{F}^{u}, \mathbf{c}_{F}^{u}}(\tau)$ and $\tilde{R}_{\mathbf{c}_{F}^{u}, \mathbf{c}_{F}^{v}}(\tau)$ are complex quantities, also the pulse correlation functions (13) and (14) are complex quantities. In the following, when talking of peaks of correlation functions, we mean the peaks of the absolute value of the complex correlation functions. Bearing in mind that $\tilde{R}_{\mathbf{c}_{F}^{u}, \mathbf{c}_{F}^{v}}(\tau)$ is a periodic function with period $\frac{k}{\Delta_{f}}, k \in \mathbb{Z}$, (9) can be rewritten as

$$
R_{s_{u}, s_{v}}(\tau) \approx \sum_{d=-\infty}^{\infty} R_{\mathbf{c}_{T}^{u}, \mathbf{c}_{T}^{v}}[d] R_{u, v}^{\text {pulse }}\left(\tau-d T_{c}\right)
$$

Eq (15) says that, when (8) holds, the autocorrelation between two doubly spread signals can be approximated by the convolution between $R_{\mathbf{c}_{T}^{u}, \mathbf{c}_{T}^{v}}[d]$ and $R_{\text {pulse }}(\tau)$.

This work focuses on the design of the frequency codes. Our aims are:

1) decorrelating the pulses (and hence the signals) assigned to different users,

2) limiting the secondary peaks of the pulses autocorrelations,

3) containing the PAPR of the signal (1) to values smaller than $3 \mathrm{~dB}$.

We set point 1 as the objective function of our optimization problem and points 2 and 3 as constraints. The limit of $3 \mathrm{~dB}$ has been chosen because it can be guaranteed using some radar pulse compression theoretical results as we will show in the next sections. Our objective is to minimise the maximum peak of $\left|R_{s_{u}, s_{v}}(\tau)\right|$ by a proper choice of $\mathbf{c}_{F}^{u}$ and $\mathbf{c}_{F}^{v}$ and thus of $\tilde{R}_{\mathbf{c}_{F}^{u}, \mathbf{c}_{F}^{v}}(\tau)$. The minimization of the maximum peak of $\left|R_{s_{u}, s_{v}}(\tau)\right|$ minimises the probability of false user detection in the acquisition process [10]. Furthermore, if Zadoff-Chu codes [13] are employed as time codes, the minimisation of the peak of the cross-correlations between signals emitted by different users optimises the whole acquisition process of the ranging signals, if we are in a scenario with low thermal noise and limited Doppler shift. Indeed, Zadoff-Chu codes have an ideal autocorrelation function and a wrong signal acquisition can only be triggered by a peak of the cross-correlation between two signals. When the same frequency code, i.e. same pulse shape, is chosen for all users, local peaks of $\left|R_{s_{u}, s_{v}}(\tau)\right|$ take place at integer multiples of $T_{c}$. These local peaks of $\left|R_{s_{u}, s_{v}}(\tau)\right|$ are equal to the maxima of $\left|R_{u, v}^{\text {pulse }}(\tau)\right|$ scaled by the values of $\left|R_{\mathbf{c}_{T}^{u}, \mathbf{c}_{T}^{v}}[d]\right|$. The above mentioned local peaks of $\left|R_{s_{u}, s_{v}}(\tau)\right|$ are highest when the pulse shape (hence frequency codes) is the same for all user, because the signal are decorrelated only by the time-domain codes, but fully correlated for the pulse shape. Choosing the same pulse for all users is the worst choice from the point of view of MAI. It is also an unnecessary choice from a system perspective. If the number of users is limited, as in the case of GNSS, choosing different optimised pulse shapes for different users becomes even a practical alternative. By minimising the maximum peak of $\left|R_{u, v}^{\text {pulse }}(\tau)\right|$, one reduces the maximum peak of $\left|R_{s_{u}, s_{v}}(\tau)\right|$. Nevertheless, because of the inter-channel interference, nothing can be stated on the relationship between the peaks of $\left|R_{u, v}^{\text {pulse }}(\tau)\right|$ and the peaks of $\left|R_{s_{u}, s_{v}}(\tau)\right|$, because the local peaks will not be necessarily located at exactly integer multiples of $T_{c}$. The secondary peak of an autocorrelation function $R(x)$ will be denoted with the notation $2^{\text {nd }} \max [R(x)]$.

\section{B. Minimal PAPR condition}

During a chip duration, the signal (1) can be considered a multitone signal:

$$
x_{u}(t)=\sum_{n=0}^{M-1} c_{F}^{u}[m-1] e^{j 2 \pi m \Delta_{f} t}=s_{u}(t),|t| \leq \frac{T_{c}}{2} .
$$

The vector $\mathbf{c}_{F}^{u}$ is the frequency code of the multitone signal. The real vectors

$$
\begin{gathered}
\boldsymbol{\rho}_{\boldsymbol{u}}=\left[\left|c_{F}^{u}[0]\right|,\left|c_{F}^{u}[1]\right|, \ldots,\left|c_{F}^{u}[M-1]\right|\right]^{\mathrm{T}} \\
\boldsymbol{\theta}_{\boldsymbol{u}}=\left[\mathrm{e}^{\mathrm{j} \arg \left\{c_{F}^{u}[0]\right\}}, \mathrm{e}^{\mathrm{j} \arg \left\{c_{F}^{u}[1]\right\}},\right. \\
\left.\ldots, \mathrm{e}^{\mathrm{j} \arg \left\{c_{F}^{u}[M-1]\right\}}\right]^{\mathrm{T}},
\end{gathered}
$$

represent the amplitudes and the phases of the subcarriers respectively. The vector $\boldsymbol{\rho}_{\boldsymbol{u}}$ is referred to as the envelope of $\mathbf{c}_{F}^{u}$. The vector $\boldsymbol{\rho}_{\boldsymbol{u}}$ determines the power distribution among the subcarriers and hence the power spectral density of the signal (16), as well as (1). The PAPR of the signal (16) is

$$
\operatorname{PAPR}_{u}\left(\boldsymbol{\rho}_{u}, \boldsymbol{\theta}_{u}\right)=\frac{\max _{t}\left\{\left|x_{u}(t)\right|^{2}\right\}}{\Delta_{f} \int_{\frac{1}{\Delta_{f}}}\left|x_{u}(t)\right|^{2} \mathrm{~d} t} \quad .
$$

The PAPR minimization problem of a multitone signal can be formulated in its most general form as: "given a vector $\boldsymbol{\rho}_{u}$, choose the vector $\boldsymbol{\theta}_{u}$ that minimises the PAPR function (19)". This problem has been tackled in many fields of communication systems [15]. One example is PAPR reduction in OFDM transmissions [16]. Some of these techniques - e.g. selected mapping and partial transmit sequence [16] - use several methods for determining a vector $\boldsymbol{\theta}_{u}$, that minimises (19) for a given vector $\rho_{u}$. These techniques are not aimed at obtaining an absolute minimum of the the function (19), but at providing a reasonably low value of (19), within reasonably short computational times and at the same time guaranteeing a sufficiently high data rate. The success of these techniques depends on the value of the vector $\rho_{u}$, and on the computational time used. In our problem we are not restricted by computational times, as the design of the spreading codes is performed off-line, and solutions more sophisticated from the computational point of view are possible. Moreover, we are also free to choose the vector $\boldsymbol{\rho}_{\boldsymbol{u}}$, i.e. the power distribution, in a convenient way. Not having to guarantee a high data link opens up new possibilities that are are of little interest for many multicarrier communication systems. The method that we propose for the PAPR minimization originates from the following idea. In general, it is not possible to state that for any given code envelope $\boldsymbol{\rho}_{\boldsymbol{u}}$ there exists a vector $\boldsymbol{\theta}_{\boldsymbol{u}}$, that minimises globally the function (19) and looking for an nonexisting global minimum can be a waste of time and resources. Hence, we propose to restrict our search to those values of $\rho_{u}$ for which we have a foreknowledge that a global maximum of the function (19) exists. In other terms, we propose to 
minimise the PAPR by a joint choice of $\boldsymbol{\rho}_{\boldsymbol{u}}$ and $\boldsymbol{\theta}_{\boldsymbol{u}}$. The relationship between the frequency code and the PAPR (19) is not an very easy, as we will show soon. Generally, designing a frequency code achieving a given PAPR is an operation that must be done by a trial and error procedure. Therefore, it is of high practical interest to work with vectors $\boldsymbol{\rho}_{\boldsymbol{u}}$ and $\boldsymbol{\theta}_{\boldsymbol{u}}$ which simplify the relationship between the frequency code and the PAPR of the signal (1), hopefully in a way to have full control on the PAPR of the ranging signal through the design of the frequency code. Both requirements - foreknowledge of the existence of a minimum of the PAPR function and simplified relationship between frequency code and PAPR - are met by a condition which we present in this section.

We indicate the aperiodic autocorrelation of $\mathbf{c}_{F}^{u}$ by

$$
r_{\mathbf{c}_{F}^{u}}[n]=\left\{\begin{array}{ll}
\sum_{k=0}^{N-n-1} c_{F}^{u}[k-n]\left(c_{F}^{u}[k]\right)^{*}, & n<0 \\
\sum_{k=0}^{N+n-1} c_{F}^{u}[k]\left(c_{F}^{u}[k+1]\right)^{*}, & n \geq 0
\end{array} .\right.
$$

The relationship between the frequency-code $\mathbf{c}_{F}^{u}$ and the instantaneous power (hence PAPR) of the corresponding multitone signal (1) is

$$
\left|x_{u}(t)\right|^{2}=r_{\mathbf{c}_{F}^{u}}[0]+2 \Re\left\{\sum_{m=1}^{M-1} r_{\mathbf{c}_{F}^{u}}[m] e^{j 2 \pi m \Delta_{f} t}\right\} .
$$

Equation (21) suggests that the instantaneous power is a multitone signal generated by the aperiodic autocorrelation coefficients of the frequency code, and thus the relationship is mathematically complex. This relationship is significantly simplified by the constraint:

$$
r_{\mathbf{c}_{F}^{u}}[m]=0, \quad m=1,2, \ldots, M-2
$$

Introducing equations (22) and (5) into (21) leads to

$$
\begin{aligned}
& \left|x_{u}(t)\right|^{2}=1+ \\
& 2\left|r_{\mathbf{c}_{F}^{u}}[\mathrm{M}-1]\right| \cos \left(2 \pi(M-1) \Delta_{f} t+\arg \left\{r_{\mathbf{c}_{F}^{u}}[M-1]\right\}\right),
\end{aligned}
$$

and thus the PAPR depends exclusively on a single parameter of the frequency code, namely the outermost coefficient of the aperiodic autocorrelation. Interestingly, condition (22) is a sufficient condition for the existence of a global minimum point for the PAPR function (19). This global minimum point is also known a-priori. In particular,

$$
\min _{\boldsymbol{\theta}_{u}}\left\{\operatorname{PAPR}_{u}\left(\boldsymbol{\rho}_{u}^{\dagger}, \boldsymbol{\theta}_{u}\right)\right\}=\operatorname{PAPR}_{u}\left(\boldsymbol{\rho}_{u}^{\dagger}, \boldsymbol{\theta}_{u}^{\dagger}\right),
$$

with $\boldsymbol{\rho}_{u}^{\dagger}$ and $\boldsymbol{\theta}_{u}^{\dagger}$ indicating the code envelope and the phase vector of any frequency code abiding condition (22).

A third effect of the condition (22) is to to limit the maximum PAPR of the signal (16) to $3 \mathrm{~dB}$ [11] for an arbitrary number of subcarriers, which is to say:

$$
\max _{\boldsymbol{\rho}_{u}^{\dagger}, \boldsymbol{\theta}_{u}^{\dagger}}\left\{\operatorname{PAPR}_{u}\left(\boldsymbol{\rho}_{u}^{\dagger}, \boldsymbol{\theta}_{u}^{\dagger}\right)\right\} \leq 3 \mathrm{~dB}
$$

Indeed, in [11] it is proven that the condition (22) is fulfilled iff $r_{\mathbf{c}_{F}^{u}}[\mathrm{M}-1]<\frac{1}{2}$.
A drawback of condition (22) is that we may be leaving out of our scope some code envelopes $\boldsymbol{\rho}_{u}$ for which the PAPR function (19) possess a global minimum without fulfilling condition (22). Indeed, we can only state that (22) is a sufficient condition for the existence of a global minimum. Nevertheless, this does not constitute a technical problem if the values of $\rho_{\boldsymbol{u}}$ fulfilling (22) can provide a choice for the spectral shape of the signal that is reasonably wide for the application at hand.

\section{Optimization problem formulation}

Let the user $u$ be assigned a signal (1) with the following properties:

- a frequency code $\mathbf{c}_{F}^{u}$ such that:

$$
4 \pi^{2} \sum_{m=0}^{M-1}\left(m-\frac{M+1}{2}\right)^{2}\left|c_{F}^{u}[m]\right|^{2}=\chi_{u}<(\mathrm{M}-3)^{2}
$$

- a secondary peak of the pulse autocorrelation equal to $\kappa_{u}$, i.e:

$$
2^{n d} \max \left[\left|R_{g}(\tau) \tilde{R}_{\mathbf{c}_{F}^{u}, \mathbf{c}_{F}^{u}}(\tau)\right|\right]=\kappa_{u}
$$

- a PAPR value equal to $\operatorname{PAPR}_{u} \leq 3 \mathrm{~dB}$.

Equation (26) is a measure of the tracking accuracy of the signal $s_{u}(t)$. We name the quantity on the left hand side of (26) equivalent MSB (EMSB) of the code $\mathbf{c}_{F}^{u}$, because this quantity determines mean-square bandwidth (MSB) [17, p.55] of a multicarrier signal with $M$ subcarriers and the central subcarrier occupying the DC frequency. The EMSB is bounded by $(\mathrm{M}-3)^{2}$, as this is the value of the EMSB when all the code energy is concentrated in the first and the last element of the frequency code $\mathbf{c}_{F}^{u}$. For simplicity $M$ is assumed always odd. Our aim is to determine the frequency spreading code $\mathbf{c}_{F}^{v}$ for the user $v$ so that the corresponding signal $s_{v}(t)$ has the following properties:

1) same tracking accuracy as $s_{u}(t)$,

2) secondary peak of the pulse autocorrelation less or equal to $\kappa_{u}$, i.e.

$$
2^{n d} \max \left[\left|R_{g}(\tau) \tilde{R}_{\mathbf{c}_{F}^{v}, \mathbf{c}_{F}^{v}}(\tau)\right|\right] \leq \kappa_{u},
$$

3) a PAPR value $\mathrm{PAPR}_{v}$ that differs from $\mathrm{PAPR}_{u}$ mostly by a $\epsilon$,

4) minimal cross-correlation peak between $s_{u}(t)$ and $s_{v}(t)$.

We can thus formulate our optimization problems with the objective function (11), constraints given by equations (5), (22), (26). Additional constraints results from the following considerations on the side-peak of the autocorrelation and on the difference between $\mathrm{PAPR}_{v}$ and $\mathrm{PAPR}_{u}$. The side-peak of the autocorrelation function is a measure of the robustness of the acquisition and of tracking process. We want that the ranging performed by the user $v$ is at least as robust as the ranging performed by user $u$. Additionally, we do not want the signals $s_{u}(t)$ and $s_{v}(t)$ to have a large difference in PAPR, because this would cause differences in the tracking accuracies, which we want to be equal by assumption. The 
tolerance on the difference of PAPR between $s_{u}(t)$ and $s_{v}(t)$ is indicated by $\epsilon$ and it depends on the HPA that it is used. All these considerations can be expressed mathematically as:

$$
\left\{\begin{array}{cl}
\underset{\mathbf{c}_{\mathbf{F}}^{\mathrm{v}}}{\operatorname{minimize}} & \max _{\tau}\left|R_{u, v}^{\text {pulse }}(\tau)\right| \\
\text { subject to } & \left\|\mathbf{c}_{F}^{v}\right\|_{2}^{2}=1 \\
& r_{\mathbf{c}_{F}^{u}}[m]=0, m=1,2, \ldots, M-2 \\
& 4 \pi^{2} \sum_{m=0}^{M-1}\left(m-\frac{M+1}{2}\right)^{2}\left|c_{F}^{v}[m]\right|^{2}=\chi_{u} \\
& 2^{n d} \max \left\{\left|R_{v, v}^{\text {pulse }}(\tau)\right|\right\} \leq \kappa_{u} \\
& \left|\operatorname{PAPR}_{u}-\operatorname{PAPR}_{v}\right| \leq \epsilon
\end{array}\right.
$$

\section{SOLUTION}

This optimization problem will be solved by inspection of all points of the feasible region. Indeed, we will show that the feasible region always contains a finite number of elements. In this section, we illustrate step-by-step how each constraint restricts the search space using the numerical values reported in Table I.

\section{A. Huffman codes}

The constraint (22) restricts our search space to the Huffman codes space [11]. In [11] it was proven that a code of length M fulfils the condition $r_{\mathbf{c}_{F}^{u}}[m]=0, m=1,2, \ldots, M-2$ (ideal-like aperiodic autocorrelation) iff its elements can be seen as the coefficients of a polynomial whose $M-1$ roots are located in a certain fashion in the complex plane(see [11] for more details). Moreover in [11] it is proved in that (22) can be fulfilled iff $\left.r_{\mathbf{c}_{F}^{u}}[M-1] \in\right] 0 ; \frac{1}{2}[$. The polynomial whose coefficients are the elements of the vector $\mathbf{c}_{F}^{v}$ in ascending order is called equivalent polynomial of $\mathbf{c}_{F}^{v}$. A Huffman code is uniquely identified by two parameters

- a binary vector $\mathbf{b}=\left[b_{0}, b_{1}, \ldots, b_{\mathrm{M}-2}\right]$, called binary generator,

- the aperiodic autocorrelation side-peak $r_{\mathbf{c}_{F}^{u}}[M-1]$.

The aperiodic autocorrelation side-peak determines the PAPR of the corresponding multitone signal, as shown in (23). The binary vector describes the location of the roots of the

\begin{tabular}{|l|l|}
\hline Parameter & Value \\
\hline$M$ & 25 \\
\hline PAPR $_{u}$ & $0.5 \mathrm{~dB}$ \\
\hline$\chi_{u}$ & 60.549 \\
\hline$\kappa_{u}$ & 0.3663 \\
\hline$\epsilon$ & $0.3 \mathrm{~dB}$ \\
\hline$\Delta_{f} T_{c}$ & 2 \\
\hline
\end{tabular}

Table I

VALUES OF THE PARAMETERS USED FOR THE SOLUTION equivalent polynomial and it determines a distinct subset of Huffman codes that is identified by the same binary generator and variable aperiodic autocorrelation side-peak. A cyclic permutation of $\mathbf{b}$ generates the same subset of distinct Huffman codes generated by $\mathbf{b}$, as it can be deduced by [18]. The variation of the parameter $r_{\mathbf{c}_{F}^{u}}[\mathrm{~m}]$ in the interval $\left[0 ; \frac{1}{2}\right]$ determines a variation of the synchronization accuracy of the corresponding two-dimensional CDMA signal, besides a variation of its PAPR: for $r_{\mathbf{c}_{F}^{u}}[m]=0$ all Huffman codes collapse to a single carrier signal at the middle of the band, i.e. minimum tracking accuracy; if $r_{\mathbf{c}_{F}^{u}}[M-1]=\frac{1}{2}$ all Huffman codes collapse to a two carriers signal at the edge of the band, i.e. maximal tracking accuracy. In the former case the EMSB is 0 and the PAPR to $0 \mathrm{~dB}$ and in the second case the EMSB is $(\mathrm{M}-3)^{2}$ and the PAPR equal to $3 \mathrm{~dB}$. Our solution is obtained by a proper choice the the parameters $\mathbf{b}$ and $r_{\mathbf{c}_{F}^{u}}[M-1]$. In the following we will explain the details.

\section{Step 1: generation of all possible distinct Huffman codes subsets}

For a start, we generate all the binary generators with this property: no binary generator of this set must be the cyclical permutation of another binary generator of the same set. This operation excludes all the binary generators that generate nondistinct Huffman codes. We call binary generators with this property distinct binary generators. For $\mathrm{M}=25$, we find 112720 distinct binary generators. Each distinct binary generator identifies a distinct Huffman code subset.

\section{Step 2: enforcing the equal EMSB constraint and unitary energy constraint}

For each binary generator we choose the value of $r_{\mathbf{c}_{F}^{u}}[M-1]$ that makes the EMSB equal to $\chi_{u}$. Experimentally it can be observed that the EMSB of a Huffman code is a monotonic function of $r_{\mathbf{c}_{F}^{u}}[M-1]$. This ensures that for each binary generator only one value of $r_{\mathbf{c}_{F}^{u}}[M-1]$ exists such that the corresponding Huffman code has a EMSB equal to $\chi_{u}$. It can also happen that there exist no value of $r_{\mathbf{c}_{F}^{u}}[M-1]$ fulfilling the condition on the EMSB. In this case we leave the binary generator out. For this reason the cardinality of the set in this step is not exactly the same as the cardinality of the set generated in the previous step, but can be smaller. All the Huffman codes are normalised to in a way that condition (5) is fulfilled. The result of this operation is the set of all existing Huffman codes of length $\mathrm{M}$ and EMSB equal to $\chi_{u}$ All two-dimensional CDMA signals (1) whose frequency code is chosen from the set generated in this step have practically the same tracking accuracy, although they spectral power density (PSD) is not necessarily the same.

Step 3: pulse autocorrelation side-peak constraint and PAPR constraint

Of all the Huffman codes generated in the previous step, we discard the ones not fulfilling the constraints of the maximum side-peak of the pulse autocorrelation and the constrain on the PAPR difference. We indicate by $\Psi\left(\chi_{u}, \kappa_{u}\right)$ the subset 
formed by all the codes generated in the previous step that that fulfil the constraint on the maximum side-peak of the pulse autocorrelation. The subset formed by all codes that fulfil the constraint on the PAPR difference is denoted by $\Xi\left(\chi_{u}, \epsilon\right)$. The cardinality of these two subsets of the set generated in step 2 depends on $\chi_{u}, \kappa_{u}$ and $\epsilon$, as well on the initial code $\mathbf{c}_{u}$. The feasible region is given by the intersection of these two subsets:

$$
\Sigma\left(\chi_{u}, \kappa_{u}\right) \cap \Xi\left(\chi_{u}, \epsilon\right)
$$

Note that if the constraints of the optimization problem on $\chi_{u}$, $\kappa_{u}$ and $\epsilon$ are too tight, this intersection may be empty. For the values used in this example and reported in Tab.I, the feasible region is a set of cardinality 9697.

\section{B. Results}

The feasible region is computed numerically as described above and the results are depicted in Fig. 1. In Fig.1a) the peaks of the cross-correlation between the pulse generated by the code $\mathbf{c}_{F}^{u}$, according to (13), and the pulse generated in an analogous by the code $\mathbf{c}_{F}^{v}$ are plotted. The frequency code $\mathbf{c}_{F}^{v}$ is kept constant, and the frequency code $\mathbf{c}_{F}^{v}$ is variable and it spans all the set generated in step 2. In Fig.1b) and Fig.1c) the effects of the constraints ( the PAPR difference and second peak of the pulse autocorrelation) on the set generated in step 2 are depicted. The border of the feasible region is marked in both graphs by a dashed line.

Let us indicate the optimal solution by $\mathbf{c}_{F}^{v_{o p t}}$. The minimum point of the objective function is $\max _{\tau}\left|\tilde{R}_{c_{F}^{u}, c_{F} v_{\text {opt }}}(\tau)\right|=$ 0.1729 . In order to have a rough measure of the significance of the results achieved, we remind the reader that Gold codes of length $N=31$ have a maximum cross-correlation peak of 0.2903. So the optimal solution is a pair of codes, $\mathbf{c}_{F}^{u}$ and $\mathbf{c}_{F}^{v_{o p t}}$ which decorrelate the signals twice as much as two Gold codes of a slightly longer length. The magnitude of the cross-correlation between the pulses assigned to the user $u$ and the optimised pulse assigned to the user $v$ is depicted in Fig.3. In Fig.2 the magnitude of the autocorrelation function of the two pulses is reported. The sharpness of the autocorrelation functions is the same because of the equivalent EMSB constraint.

Finally, we depict in Fig.4 the power spectral density of the signals assigned to user $u$ and the optimised signal assigned to then user $v$.

\section{CONCLUSIONS}

In this paper we have proposed a spreading code design for an asynchronous two-dimensional CDMA system. The objective was to design the frequency domain-spreading codes that minimise the MAI between the signals assigned to two users $u$ and $v$ and keep the PAPR below $3 \mathrm{~dB}$. Other constraints have been placed on the secondary peak of the pulse autocorrelation functions and on the difference between the PAPRs of the signals relative to the two different users. a)
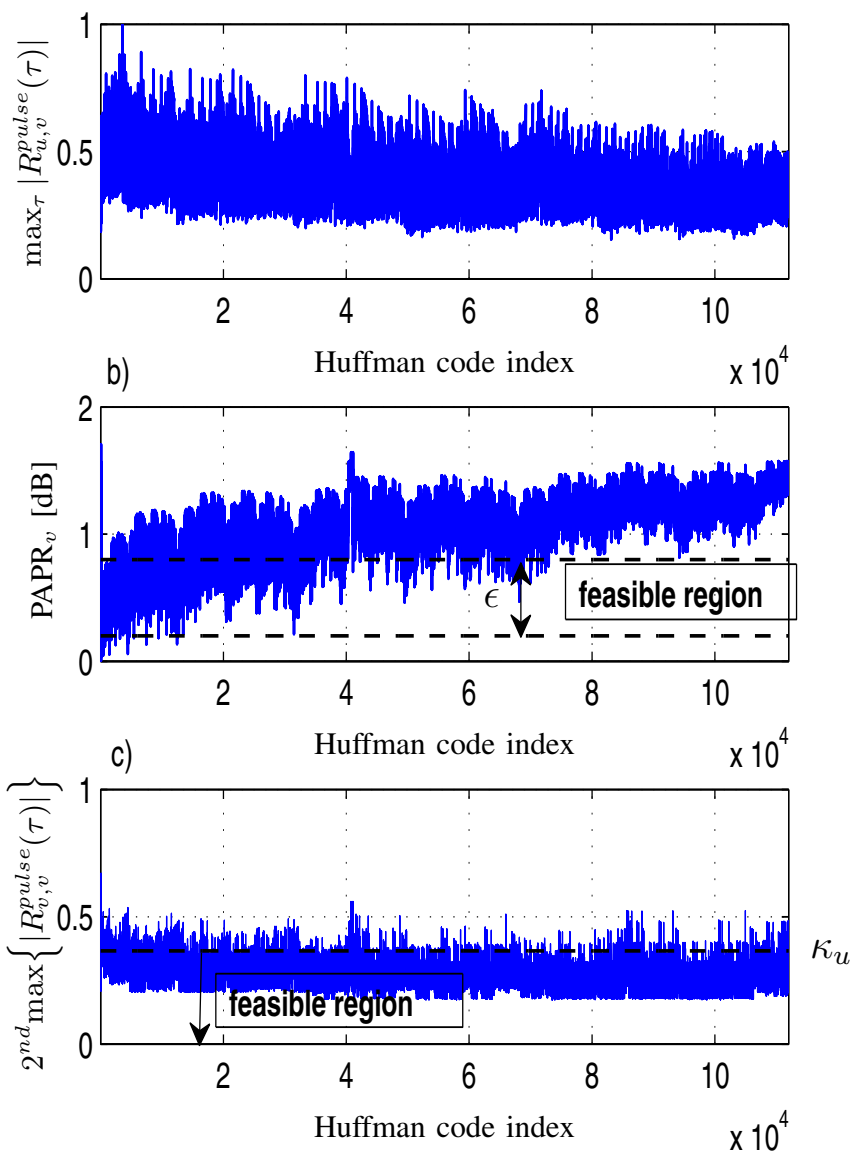

Fig. 1. a) Objective function evaluated for each element of the set generated in step 2, b) corresponding PAPR of the user $v$ in $\mathrm{dB}, \mathrm{c}$ ) Corresponding Autocorrelation side-peak of the signal of the user $v$.

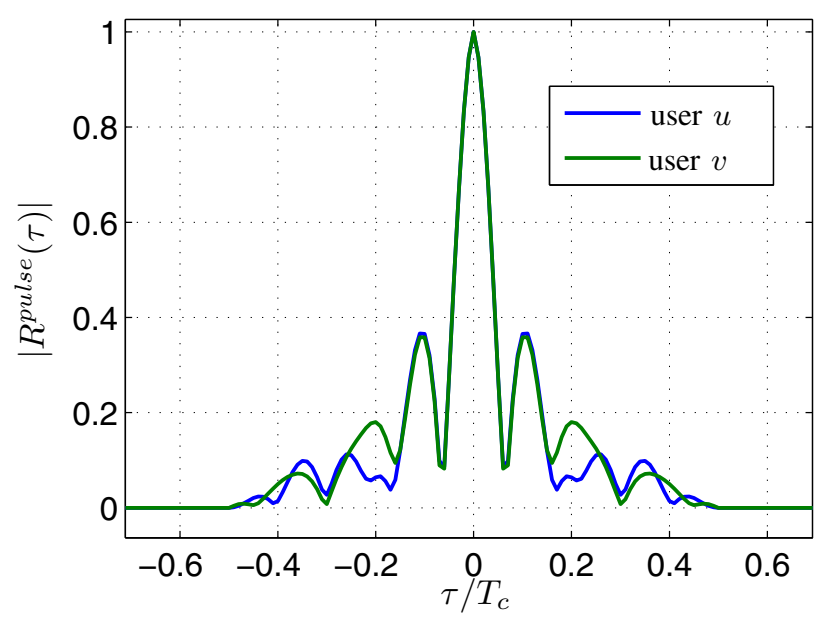

Fig. 2. Absolute value of the pulses autocorrelation functions.

The method is valid for an arbitrary number of subcarriers and it is based on some codes (Huffman codes) that were developed several decades ago for radar pulse compression. The optimization problem could be solved by inspection of all 


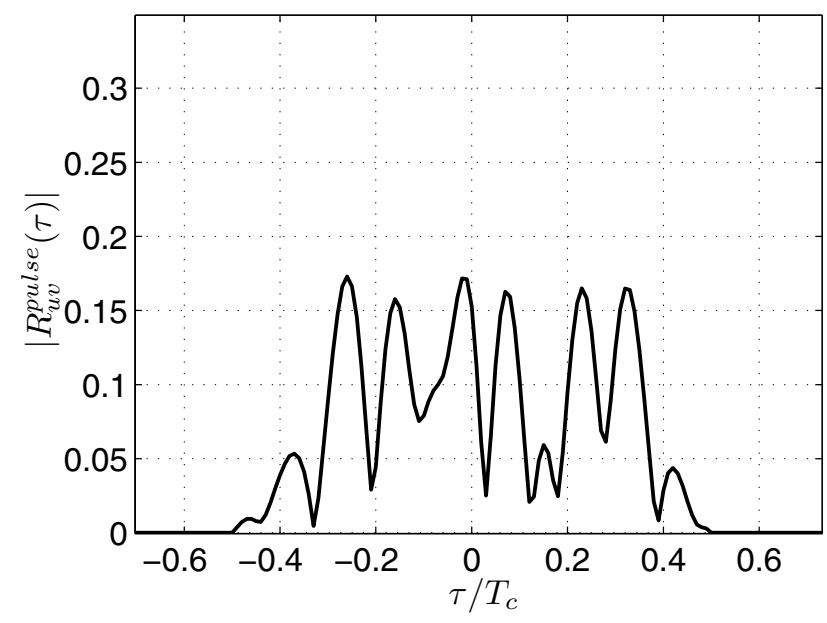

Fig. 3. Absolute value of the pulses cross-correlation function.

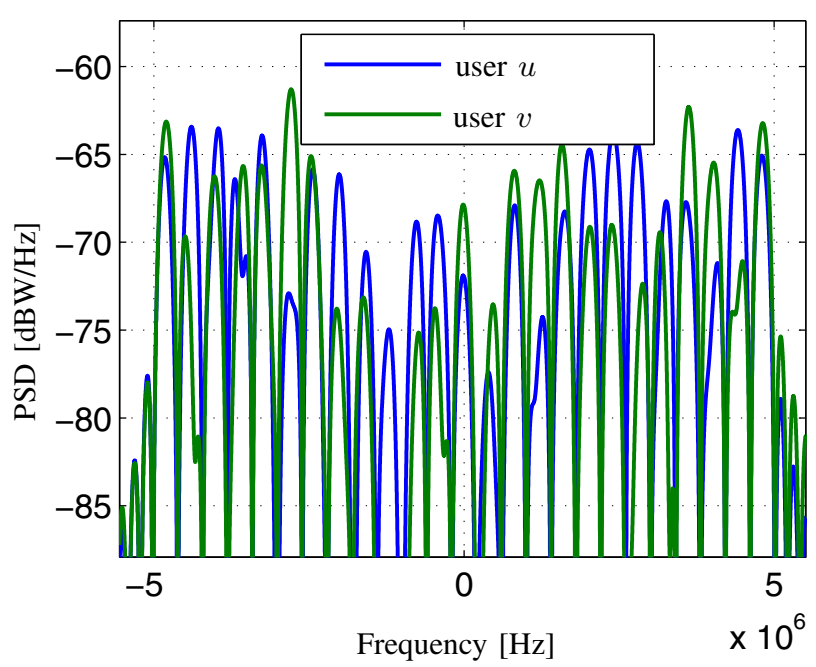

Fig. 4. Power spectral density of the optimal solution (user $v$ ) in comparison with the PSD of the signal assigned to the user $u$.

solutions, because the feasible region is a finite, discrete set. A shortcoming of our work is that the condition (22) excludes some frequency codes that may still have a PAPR lower than $3 \mathrm{~dB}$ and which may also yield lower peaks of the spectral cross-correlation function.

\section{REFERENCES}

[1] S. Hara and R.Prasad, "Overview of multicarrier CDMA," IEEE Communications Magazine, vol. 35, no. 12, p. 126133, December 1997.

[2] L. Yang and L. Hanzo, "Multicarrier DS-CDMA: A Multiple Access Scheme for Ubiquitous Broadband Wireless Communications," IEEE Communication magazine, pp. 116-124, October 2003.

[3] N. Yee, J. P. Linnartz, and G. Fettweis, "Multicarrier CDMA in indoor wireless radio networks," in Proc. PIMRC, Yokohama, Yokohama, Japan, May 1993, p. pp. D1.3.1D1.3.5.

[4] C.-M. Yang, P.-H. Lin, and G.-C. Yang, "2D orthogonal spreading codes for multicarrier DS-CDMA systems," in IEEE International Conference on Communications, 2003 (ICC '03), Anchorage, AK, May 2003, pp. $3277-3281$ vol.5.

[5] C.-W. Chang, "An Interference-Avoidance Code Assignment Strategy for the Hierarchical Two-Dimensional-Spread MC-DS-CDMA System: A Prototype of Cognitive Radio Femtocell System," IEEE Trans. on Vehicular Technology, vol. 61, no. 1, November 2012.
[6] L. Wang, C. Chang, and H. Huang, "An Interference Avoidance Code Assignment Strategy for Downlink Multi-Rate MC-DS-CDMA with TFDomain Spreading," IEEE Trans. on Wireless Communications, vol. 6, no. 7, July 2007.

[7] Y. Zhou, T.-S. Ng, J. Wang, K. Higuchi, and M.Sawahashi, "OFCDM: A Promising Broadband Wireless Access Technique," IEEE Communications Magazine, vol. 46, no. 3, pp. 38-49, March 2008.

[8] Q.Shi and M.Latva-aho, "Performance Analysis of MC-CDMA in Rayleigh fading channels with correlated envelopes and phases," IEE Proceedings in Communications, vol. 150, no. 3, pp. 214-220, June 2003.

[9] D.Sarawate, "Bounds on Crosscorrelation and Autocorrelation of Sequences (corresp.)," IEEE Trans. Inform. Theory, vol. 25, no. 6, pp. 720-724, May 1979.

[10] M. Pursley, "Performance Evaluation for Phase-Coded Spread-Spectrum Multiple-Access Communication-Part I: System Analysis," IEEE Trans. on Communications, vol. 25, no. 8, pp. 795-799, August 1977.

[11] D. A. Huffman, "The generation of impulse equivalent pulse trains," IRE. Trans. Inform. Theory, vol. ITS, no. 5, pp. 10-16, 1962.

[12] H. G. Feichtinger and e. T. Strohmer, Gabor Analysis and Algorithms:Theory and Applications. Boston: Birkhauser, 1998.

[13] D. C. Chu, "Polyphase codes with good periodic correlation properties," IEEE Trans. Inform. Theory, vol. IT-18, pp. 531-532, July 1972.

[14] X. Li, "Statistical Analyis and Reduction of Multiple Access Interference in MC-CDMA Systems," Ph.D. dissertation, Queensland University of Technology, 2008.

[15] D. R. Gimlin, "On Minimizing the Peak-to-Average Power Ratio for the Sum of N Sinusoids," IEEE Trans. on Communiations, vol. 41, no. 4, April 1993.

[16] S. Han and J. H. Lee, "An Overview of Peak-to-average Power Ratio Reduction Techniques for Multicarrier Transmission," IEEE Wireless Communication Magazine, pp. pp. 56-65, April 2005.

[17] S. M. Kay, Fundamentals of Statistical Signal Processing, Volume I estimation theory. Prentice Hall, 1998.

[18] L. Bomer and M. Antweiler, "Long energy efficient Huffman sequences," in International Conference on Acoustics, Speech, and Signal Processing ICASSP-91, April 1991, pp. 2905-2908. 